\title{
A GENERALIZED BESSEL INEQUALITY
}

\author{
ELENA PRESTINI
}

\begin{abstract}
To any pair of diadic intervals $[I, \omega]$ with $I \subseteq[0,1)$ and $\omega=\left[N_{\omega}, N_{\omega}\right.$ $\left.+|I|^{-1}\right)$ we associate the function $u_{I I, \omega]}(x)=|I|^{-1 / 2} e^{i N_{\omega} x} \chi_{V}(x)$. In this paper we will give a condition under which a collection $B$ of such pairs satisfies the inequality $\Sigma_{[I, \omega] \in B}\left|\left\langle u_{[I, \omega]}(x), f(x)\right\rangle\right|^{2}<200\|f\|_{2}^{2}$ for any $f$ in $L_{2}[0,1)$.
\end{abstract}

Introduction. In this paper we prove an inequality of Bessel type for functions in $L_{2}[0,1)$ with respect to a class of "almost orthogonal" systems. These systems can be described as collections of functions obtained by restricting to diadic intervals of length $2^{-n}$ characters whose frequency is a multiple of $2^{n}(n>0)$. The "almost orthogonality" of the systems is given by an interesting property of separation on the supports of the characters involved or on their frequency.

These systems, which might seem not that natural at a first glance, turned out to be very useful in studying the problem of pointwise convergence of Fourier series [4]. We will say more about this at the end of the paper.

Preliminary lemmas. In what follows we write $e^{i x}$ instead of $\exp (2 \pi i x)$. The proof of the result is based on the well-known

Cotlar's Lemma. Suppose that the operators $L_{1}, L_{2}, \ldots, L_{N}$ on a Hilbert space $H$ satisfy the "orthogonality conditions"

$$
\left\|L_{k}^{*} L_{r}\right\| \leqslant C(k-r), \quad\left\|L_{k} L_{r}^{*}\right\| \leqslant C(k-r)
$$

where $\|\cdot\|$ denotes the operator norm and $\sum_{j=-\infty}^{+\infty} C(j)^{1 / 2}<A$. Then $\left\|\sum_{k=1}^{N} L_{k}\right\| \leqslant$ A.

For the proof see [1].

We shall also need the following

LEMMA. Suppose $L$ is an integer not smaller than 1 and there is given a sequence of integers $\left\{n_{h}\right\}$ such that for any two of them, $n_{h_{1}}$ and $n_{h_{2}},\left|n_{h_{1}}-n_{h_{2}}\right|>2 L$. Consider the following operator defined on $l_{2}$

$$
H_{L}\left\{b_{n}\right\}(m)=\sum_{|n-m|>L} \frac{b_{n}}{n-m} .
$$

Then for any sequence $\left\{a_{n}\right\} \in l_{2}$ such that $a_{n}=0$ whenever $n$ belongs to the set $\cup_{h}\left\{m \in Z:\left|m-n_{h}\right|<2 L\right\}$ we have

$$
\sum_{h}\left|H_{L}\left\{a_{n}\right\}\left(n_{h}\right)\right|^{2} \leqslant 20 / L \sum_{n=-\infty}^{+\infty}\left|a_{n}\right|^{2} .
$$

Received by the editors January 24, 1980.

1980 Mathematics Subject Classification. Primary 42A20, 42 A24.

(c) 1981 American Mathematical Society $0002-9939 / 81 / 0000-0423 / \$ 02.00$ 
Proof. We may assume $a_{n} \geqslant 0$ for every $n$. We fix $n_{h}$ and without loss of generality we assume it to be 0 . Then we write with obvious notations

$$
\begin{aligned}
& H_{L}\left\{a_{n}\right\}(0)=\sum_{n>L} \frac{a_{n}}{n}+\sum_{n<-L} \frac{a_{n}}{n}=B_{0}-A_{0}, \\
& H_{L}\left\{a_{n}\right\}(j)=\sum_{n-j>L} \frac{a_{n}}{n-j}+\sum_{n-j<-L} \frac{a_{n}}{n-j}=B_{j}-A_{j} .
\end{aligned}
$$

First suppose $B_{0} \geqslant A_{0}$. Then, since $a_{n}=0$ for $n \in(-2 L, 2 L)$ and $a_{n}$ is nonnegative, we have $B_{j} \geqslant B_{0} \geqslant A_{0} \geqslant A_{j}$ for $j=1, \ldots, L$. Now suppose $B_{0}<A_{0}$. In this case $A_{j} \geqslant A_{0} \geqslant B_{0} \geqslant B_{j}$ for $j=-L, \ldots,-1$. In any case

$$
\left|H_{L}\left\{a_{n}\right\}(0)\right|^{2} \leqslant 1 / L \sum_{j=-L}^{+L}\left|H_{L}\left\{a_{n}\right\}(j)\right|^{2}
$$

Hence because of the "distance property" of the $n_{h}$ 's and of the fact that $H_{L}$ is a bounded operator on $l_{2}$, we have

$$
\sum_{h}\left|H_{L}\left\{a_{n}\right\}\left(n_{h}\right)\right|^{2} \leqslant 1 / L \sum_{j=-\infty}^{+\infty}\left|H_{L}\left\{a_{n}\right\}(j)\right|^{2} \leqslant 10 / L \sum_{n=-\infty}^{+\infty}\left|a_{n}\right|^{2} .
$$

The result.

THEOREM. Suppose there is given a collection $B$ of pairs $p=\left[I_{k}^{j}, \omega_{k}^{h}\right]$ where $I_{k}^{j}=\left[j 2^{-k},(j+1) 2^{-k}\right), j=0, \ldots,\left(2^{k}-1\right), k$ a nonnegative integer and $\omega_{k}^{h}=\left[h 2^{k}\right.$, $\left.(h+1) 2^{k}\right), h$ any integer, with the following property

$$
\begin{aligned}
& I_{k}^{j} \cap I_{k^{\prime}}^{i^{\prime}}=\varnothing \text { or } \\
& I_{k}^{j} \cap I_{k^{\prime}}^{j^{\prime}} \neq \varnothing \quad \text { and } \operatorname{dist}\left(\omega_{k}^{h}, \omega_{k^{\prime}}^{h^{\prime}}\right)>\max \left(\left|\omega_{k}^{h}\right|^{a},\left|\omega_{k^{\prime}}^{h^{\prime}}\right|^{a}\right)
\end{aligned}
$$

for some $a>0$ and for any two pairs $\left[I_{k}^{j}, \omega_{k}^{h}\right],\left[I_{k^{\prime}}^{j^{\prime}}, \omega_{k^{\prime}}^{h^{\prime}}\right]$ in B. For $f$ in $L_{2}[0,1)$ and for any pair $[I, \omega] \in B$ with $\omega=\left[N_{\omega}, N_{\omega}+2^{k}\right)$ define

$$
c_{[I, \omega]}=1 /|I| \int_{I} f(x) e^{-i N_{\omega} x} d x
$$

Then the inequality

$$
\sum_{[I, \omega] \in B}\left|c_{[I, \omega]}\right|^{2}|I| \leqslant 200\|f\|_{2}^{2}
$$

holds:

(i) for $a>2$,

(ii) for $a>1$ as well, if we further assume that for each $I_{k}^{j}$ there exists at most one pair $\left[I_{k}^{j}, \omega\right]$ in B. For $a=1$ this additional assumption is not enough to guarantee (1).

Proof. We denote by $T_{[I, \omega]}$ the operator that acts on $f \in L_{2}[0,1)$ as follows $T_{[I, \omega]} f(x)=\left.c_{[I, \omega]} e^{i N_{\omega} x}\right|_{I}$. Clearly $T_{p}$ is selfadjoint and $T_{p}^{*} T_{p}=T_{p}$. Now we denote by $R_{k}$ the subcollection of pairs of $B$ such that $|I|=2^{-k}$, by $T_{k} f$ the sum $\Sigma_{p \in R_{k}} T_{p} f$ 
and we observe that

$$
\begin{aligned}
\sum_{[I, \omega] \in B}\left|c_{[I, \omega]}\right|^{2}|I| & =\sum_{p \in B}\left\|T_{p} \dot{f}\right\|_{2}^{2}=\sum_{p \in B}\left(T_{p} f, T_{p} f\right) \\
& =\sum_{p \in B}\left(f, T_{p}^{*} T_{p} f\right)=\sum_{p \in B}\left(f, T_{p} f\right) \\
& =\left(f, \sum_{k=0}^{+\infty} T_{k} f\right)<\|f\|_{2}\left\|\sum_{k=0}^{+\infty} T_{k} f\right\|_{2}
\end{aligned}
$$

We shall prove, using Cotlar's lemma, that $\left\|\sum_{k=0}^{+\infty} T_{k} f\right\|_{2}^{2}<200\|f\|_{2}^{2}$. Using property $(P)$, we have that

$$
\left\|T_{k} f\right\|_{2}^{2}=\sum_{p \in R_{k}}\left\|T_{p} f\right\|_{2}^{2} \leqslant \sum_{|I|=2^{-k}}\left\|\left.f\right|_{I}\right\|_{2}^{2} \leqslant\|f\|_{2}^{2}
$$

for every $k$. Hence $\left\|T_{k}\right\| \leqslant 1, k \geqslant 0$. Now we just have to estimate $\left\|T_{r} T_{k}\right\|$ since $\left\|T_{r}^{*} T_{k}\right\|=\left\|T_{r} T_{k}^{*}\right\|=\left\|T_{r} T_{k}\right\|$. We start assuming $r>k$. Then in the sum $T_{r} T_{k} f=$ $\sum_{p^{\prime} \in R_{r}} T_{p^{\prime}}\left(\sum_{p \in R_{k}} T_{p} f\right), T_{p^{\prime}} T_{p}$ is different from the zero operator only in the case that $I^{\prime} \subset I$, with $p^{\prime}=\left[I^{\prime}, \omega^{\prime}\right]$ and $p=[I, \omega]$. Now we observe that

$$
\left\|T_{r} T_{k} f\right\|_{2}^{2}=\sum_{\left|I^{\prime}\right|=2^{-r}}\left\|\sum_{\substack{I \supset I^{\prime} \\ p=[I, \omega] \in R_{k} \\ p^{\prime}=\left[I^{\prime}, \omega^{\prime}\right] \in R_{r}}} T_{p^{\prime}} T_{p} f\right\|_{2}^{2} .
$$

Fix $I^{\prime}=I_{0}^{\prime}=\left[x_{I_{0}}^{1}, x_{I_{0}^{\prime}}^{2}\right)$ and consider all the pairs belonging to $R_{r}$ such that their first element is $I_{0}^{\prime}$. Observe also that there exists just one $I$ with $|I|=2^{-k}$ such that $I \supset I_{0}^{\prime}$. Call it $I_{0}$. Then

$$
\begin{aligned}
& \left\|\sum_{\substack{p=\left[I_{0}, \omega\right] \in R_{k} \\
p^{\prime}=\left[I_{0}^{\prime}, \omega^{\prime}\right] \in R_{r}}} T_{p^{\prime}} T_{p} f\right\|_{2}^{2}=\sum_{\substack{\omega^{\prime} \\
p^{\prime}=\left[I_{0}^{\prime}, \omega^{\prime}\right] \in R_{r}}}\left\|_{p=\left[I_{0}, \omega\right] \in R_{k}} T_{p^{\prime}} T_{p} f\right\|_{2}^{2} \\
& =\left.\left.\sum_{N_{\omega^{\prime}}}\left|\sum_{\omega}\right| I_{0}^{\prime}\right|^{-1} c_{\left[I_{0} \omega\right]} \int_{I_{0}^{\prime}} e^{i\left(N_{\omega}-N_{\omega^{\prime}}\right) x} d x\right|^{2} 2^{-r} \\
& =2^{r} \sum_{N_{\omega^{\prime}}}\left|\sum_{\omega} c_{\left[I_{0}, \omega\right]} \frac{e^{i\left(N_{\omega}-N_{\omega^{\prime}}\right) x_{I_{0}^{\prime}}^{2}-e^{i\left(N_{\omega}-N_{\omega^{\prime}}\right) x_{i_{0}^{\prime}}^{1}}}}{N_{\omega}-N_{\omega^{\prime}}}\right|^{2}
\end{aligned}
$$

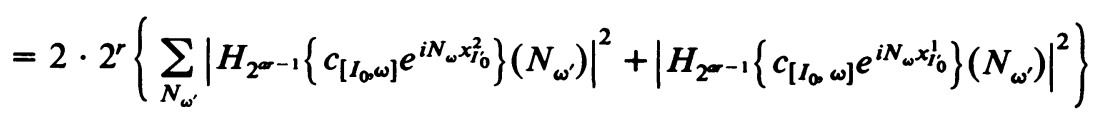

$$
\begin{aligned}
& \leqslant 200 \cdot 2^{r} \cdot 2^{-a r} \sum_{\omega}\left|c_{\left[I_{0, \omega}\right)}\right|^{2} \\
& \leqslant 200 \cdot 2^{-(a-1) r} \cdot 2^{k}\left\|\left.f\right|_{I_{0}}\right\|_{2}^{2} \text {, by the lemma. }
\end{aligned}
$$

If one frequency interval at most is associated to each $I_{k}^{j}$ then no summation on $N_{\omega^{\prime}}$ and on $\omega$ appears in the above computations and the upper bound is easily seen to become $2^{-(2 a-1) r} \cdot 2^{k}\left\|\left.f\right|_{I_{0}}\right\|_{2}^{2}$. Now we go back to (2). Using (3) and the fact that the 
number of $I^{\prime}$ contained in $I_{0}$ is $2^{r-k}$ we obtain

$$
\begin{aligned}
\left\|T_{r} T_{k} f\right\|_{2}^{2} & \leqslant 200 \cdot 2^{r-k} \sum_{|I|=2^{-k}} 2^{k} \cdot 2^{-(a-1) r}\left\|\left.f\right|_{I}\right\|_{2}^{2} \\
& \leqslant 200 \cdot 2^{-(a-2) r}\|f\|_{2}^{2}
\end{aligned}
$$

and $\left\|T_{r} T_{k} f\right\|_{2}^{2} 2^{-(2 a-2) r}\|f\|_{2}^{2}$ with the additional assumption contained in (ii). Now still assuming $r>k$ we observe that $\left\|T_{k} T_{r}\right\|=\left\|\left(T_{k} T_{r}\right)^{*}\right\|=\left\|T_{r} T_{k}\right\|<2^{-(a-2) r / 2}$. Hence Cotlar's lemma gives the inequality we want, when $a>2$ in the case (i) and similarly when $a>1$ in the case (ii).

Next we give a counterexample (due to F. Ricci) for the case $a<1$. Suppose that the collection of pairs is $B=\left\{\left[I_{k}^{j}, \omega_{k}\right], I_{k}^{j}=\left[j 2^{-k},(j+1) 2^{-k}\right), j=0, \ldots,\left(2^{k}-1\right)\right.$, $\left.\omega_{k}=\left[3 \cdot 2^{k}, 2^{k+2}\right), k \geqslant 0\right\}$. We choose $f=e^{-i x}$. We write $c_{k}^{j}=c_{\left[I_{k}^{\prime}, \omega_{k}\right]}$. Then

$$
\left|c_{k}^{j}\right|=\left|2^{k} \int_{j 2^{-k}}^{(j+1) 2^{-k}} e^{-i x} e^{-i 3 \cdot 2^{k x}} d x\right|=\frac{\left|e^{-i\left(3+2^{-k}\right)}-1\right|}{3+2^{-k}} .
$$

Hence

$$
\sum_{k=0}^{+\infty} \sum_{j=0}^{2^{k}-1}\left|c_{k}^{j}\right|^{2} 2^{-k}=\sum_{k=0}^{+\infty} \frac{\left|e^{-i\left(3+2^{-k}\right)}-1\right|^{2}}{\left(3+2^{-k}\right)^{2}}
$$

which diverges. This shows that (1) does not hold for $a=1$.

\section{Comments.}

1. The results do not appear to be typical of the exponentials. They depend instead on the "amount of orthogonality" of the systems. For instance if we substitute the exponentials with Walsh functions it is easy to see that the corresponding systems are orthogonal for all values of $a>0$. Actually more is true. If $I_{k}^{j} \cap I_{k^{\prime}}^{j^{\prime}} \neq \varnothing$ it is enough to require $\omega_{k}^{h} \cap \omega_{k^{\prime}}^{h^{\prime}}=\varnothing$ in the separation property to have orthogonal systems. This trivially implies (1). (See [3] for an instance of a collection of functions closely related to ours.)

2. In [5] the basic estimate which is needed is an inequality like (1) in the case $a=1$. (See [2], [4] where orthogonal operators related to this case appear.) While such an inequality is true for Walsh functions, we know that it is not true in general for the exponentials. In [5] another kind of conditions (basically a control on the number of rows of operators involved) guarantees that the same Bessel type inequality is true.

\section{REFERENCES}

1. M. Cotlar, A unified theory of Hilbert transforms and ergodic theorems, Rev. Math. Cuyana 1 (1955), 105-167.

2. J. A. Gosselin, On the convergence of Walsh-Fourier series for $L^{2}(0,1)$, Adv. in Math. Suppl. Studies 4 (1979), 223-232.

3. R. A. Hunt, Ensemble exceptionels en analyse, Actes du Congrès International des Mathématiciens (Nice, 1970), vol. 2, Gauthier-Villars, Paris, 1971, pp. 655-661.

4. C. Fefferman, Pointwise convergence of Fourier series, Ann. of Math. (2) 3 (1973), 551-572.

5. E. Prestini, On the two proofs of pointwise convergence of Fourier series, Amer. J. Math. (to appear).

Istituto di Matematica, Università di Milano, Milano, Italia 